\title{
Power-Efficient Routing Mechanism for ODMA Systems
}

\author{
Ray-Guang Cheng, Member, IEEE, Shin-Ming Cheng, and Phone Lin, Senior Member, IEEE
}

\begin{abstract}
Opportunity driven multiple access (ODMA), a cellular multihop method proposed for Universal Mobile Telecommunications Systems, potentially allows reduction in power consumption of user equipment (UE), extending Node B's coverage and supporting higher user data rate. However, ODMA requires extra power for discovering relaying nodes and introduces additional transmission latency in data transfer. This paper offers enlightenment to these ODMA implementation problems. A power-efficient routing (PER) mechanism is proposed to identify a minimum-power path for ODMA communication. Prior to the route (or path) discovery, the PER mechanism utilizes an analytical solution to estimate the total power and number of intermediate UEs required in the minimum-power path. With the estimation, route discovery procedures originating from nonattainable ODMA requests can be prevented. For those attainable ODMA requests that require a route discovery procedure to locate intermediate UEs, the PER mechanism further provides a method to set the transmission power and maximum hop count. Hence, the power consumption of each UE during route discovery is significantly reduced. Simulation results coincided with the analysis, and the results demonstrate the performance improvement of PER over dynamic source routing.
\end{abstract}

Index Terms-Opportunity driven multiple access (ODMA), power-efficient routing (PER).

\section{INTRODUCTION}

$\mathbf{O}$ PPORTUNITY driven multiple access (ODMA) is an ad hoc multihop relaying protocol [1] considered by the third-generation partnership project (3GPP) working group [2]. Although it has now been dropped to achieve a finalized standard as a result of concerns over complexity, battery life of users on standby, and signaling overhead issues, ODMA remains an attractive prospect for future mobile communication systems [3]. The advantages of ODMA include 1) potentially reduced transmission power; 2) possibly enhanced coverage; 3) increased capacity under certain circumstances; and 4) a

Manuscript received January 7, 2005; revised August 2, 2005, November 3, 2005, and November 11, 2005. This work was supported by the Chung-Shan Institute of Science and Technology, Taiwan, under Contract BC93203P; and in part by the National Science Council (NSC), Taiwan, R.O.C., under Contracts NSC 94-2219-E-011-005, NSC 94-2213-E-002-083, NSC 94-2213-E-002-090, and NSC 94-2627-E-002-001; the Ministry of Economic Affairs (MOEA), Taiwan, R.O.C., under Contract 93-EC-17-A-05-S1-0017; the Computer and Communications Researches Labs/Industrial Technology Research Institute (CCL/ITRL); Chunghwa Telecom Labs; Telcordia Applied Research Center; the Taiwan Network Information Center (TWNIC); and Microsoft Corporation, Taiwan, R.O.C. The review of this paper was coordinated by Dr. W. Zhuang.

R.-G. Cheng is with the Department of Electronic Engineering, National Taiwan University of Science and Technology, Taipei 106, Taiwan, R.O.C. (e-mail: crg@et.ntust.edu.tw).

S.-M. Cheng and P. Lin are with the Department of Computer Science and Information Engineering, National Taiwan University, Taipei 106, Taiwan, R.O.C. (e-mail: shimi@pcs.csie.ntu.edu.tw.; plin@csie.ntu.edu.tw).

Digital Object Identifier 10.1109/TVT.2006.877457 greater tradeoff between quality-of-service (QoS) and capacity in the extended coverage areas [3], [4].

In ODMA, user data are exchanged between a sending mobile station [also known as user equipment (UE) in Universal Mobile Telecommunications System (UMTS)] and the base station (called Node B in UMTS) by being relayed through other intermediate UEs. The sending UE should establish a path through the intermediate UEs to Node B prior to data exchange, which introduces additional signaling overhead and thus results in extra power consumption for certain UEs. Hence, a good routing mechanism with low signaling overhead would be essential while realizing ODMA. Unlike mobile ad hoc networks (MANETs) [5] where communication generally occurs between any pair of nodes through several mobile relaying nodes, a sending UE in ODMA always exchanges data with Node B by utilizing nonmobile or low-mobility intermediate UEs [4]. Moreover, most of the nodes in MANET cannot directly communicate with each other due to the limited transmission power. However, all UEs in ODMA can communicate with Node B directly. Hence, existing routing methods proposed for MANET may not be directly applicable to ODMA.

Several power-aware routing methods [6]-[10] have been proposed for MANET and ODMA cellular networks. Most of the proposed methods are developed out of the dynamic source routing (DSR) protocol [11] and the ad hoc on-demand distance vector (AODV) routing protocol [12]. In DSR and AODV, the source node initiates a route discovery procedure by flooding a route request (RREQ) packet its surrounding nodes. The RREQ is always forwarded by intermediate nodes until the destination node is reached. The destination node sends back a route reply (RREP) packet carrying the power metrics of the selected path(s) to the source node. A minimum-power path is then identified based on the collected metrics. The power consumption of nodes in MANET was first considered by Singh et al. [6] in their routing method. Chang and Tassiulas [7] considered the residual power of UEs in their energy-efficient routing algorithm. Rodoplu and Meng [8] proposed a position-based routing method for mobile wireless networks. This method constructed a position-based sparse graph for all communication links connecting mobile nodes and then derived a minimumpower routing topology from the graph. Wattenhofer et al. [9] proposed a distributed topology control algorithm for MANET. Using the directional antenna technology, each UE constructed a communication graph, removed the nonefficient edges from the graph, and derived a minimum-power routing topology. The Vodafone Group [10] proposed an ODMA routing procedure where the given local and end-to-end connectivity information was utilized to construct the routing path. 
Three significant assumptions are made in the above proactive routing approaches. The first assumption is that each node retains the up-to-date location information and/or power metrics of the other nodes. This assumption may be effective in MANET but is not suitable for mobile cellular networks that enable the discontinuous reception (DRX) function. With DRX, a UE is in sleep mode for most of the time to save power and periodically wakes up to gather system information. Hence, each UE in a mobile cellular network cannot have up-to-date information of other UEs because all information would be obsolete after returning to sleep mode. This assumption may be relieved by employing reactive routing approaches [13]. However, existing reactive routing approaches rely on a route discovery procedure to obtain the other UEs' information. Hence, some routing control messages may be wasted on processing nonattainable ODMA requests (i.e., the power or latency requirement for those requests cannot be attained by utilizing the ODMA technology). The second assumption is that the extra power used by RREQ signaling is ignored. Therefore, RREQ in MANET is always flooded among UEs with UE's maximum transmission power and without hop count limitation. UE's transmission power can be up to several watts in a mobile cellular network, which cannot be neglected. The third assumption is that the power metric only considers the path loss between two adjacent UEs but neglects the power consumed by UEs' receivers.

This paper presents a power-efficient routing (PER) mechanism and identifies parameters required to discover a minimum-power path for ODMA communication. Different to the existing reactive routing approaches, the PER mechanism utilizes an analytical solution to estimate the total power (i.e., including the power consumed by UEs' receivers) and number of intermediate UEs required in the minimum-power path prior to route discovery. With the prediction, route discovery procedures originating from nonattainable ODMA requests can be prevented. For those attainable ODMA requests that require a route discovery procedure to locate intermediate UEs, the PER mechanism further provides a method to set the transmission power and maximum hop count when forwarding RREQ. With these settings, the power consumption of each UE during the route discovery can be significantly reduced.

The rest of this paper is organized as follows. Section II proposes the PER mechanism and discusses its key parameters and the effect of the parameters on system performance. Section III investigates the performance of the PER mechanism via numerical analysis and simulation. Conclusions are finally drawn in Section IV.

\section{PER MECHANISM}

A time division duplex (TDD)-ODMA network [3] comprising of Node B and several nonmobile ODMA-enabled UEs, which are identified by their user-specific identities (ODMA_IDs), is considered herein. It is assumed that Node B may allocate dedicate timeslots for the ODMA communication to minimize the power warfare problem [3] among ODMA and non-ODMA UEs. To simplify our description, we use the term "UE" to denote an ODMA-enable UE. In an ODMA transmission, the UEs are categorized into three types, namely 1) SendingUE; 2) BackerUE; and 3) RelayUE. A SendingUE originates the ODMA transmission. The other UEs that participate in the ODMA route discovery within the cell are BackerUEs. Among these BackerUEs, some will be identified as RelayUEs, which are responsible for relaying data packets between the SendingUE and Node B. Note that UEs that do not have sufficient residual power may optionally disable some ODMA functionalities to reduce unnecessary power consumption.

This study considers three power consumption modes of the UE, including sleep (SLP), receive (RX), and transmit (TX). In SLP mode, the UE consumes the least amount of power for running a timer. In RX mode, the receiver is turned on, and the UE can receive data from other UEs and Node B. In TX mode, the transmitter is turned on, and the UE can adjust its transmission power while transmitting data. The parameters used in the PER mechanism are defined as follows.

- $P_{\text {ref }}$ and $\alpha P_{\text {ref }}$ are the minimum and maximum powers consumed by the UE in TX mode, respectively. $\beta P_{\text {ref }}$ is the average power consumed by the UE in RX mode. $\gamma P_{\text {ref }}$ is the average power consumed by the UE in SLP mode. $\alpha, \beta$, and $\gamma$ are constant numbers and with the relationship $\alpha>1>\beta \gg \gamma>0$ [1].

- $P_{\text {TX_RDP }}$ is the transmission power consumed by the UE when forwarding RREQ in the "path discovery phase."

- $N_{\max }$ is the maximum hop count that an RREQ can traverse in the "path discovery phase." $N_{\text {opt }}$ is the number of RelayUEs required in an optimal path. The optimal path exists when the RelayUEs can be found at any location within a cell.

- $P_{\text {total }, i}$ is the total power required by the $i$ th path discovered in the "path discovery phase." $P_{\mathrm{opt}}$ is the total power required in the optimal path. Note that $P_{\text {total }, i} \geq P_{\text {opt }}$.

- $P_{\text {ini }}$ is the transmission power consumed by the SendingUE to send the ODMA service request.

The PER mechanism consists of three phases, namely 1) access phase; 2) path discovery phase; and 3) path setup phase. In access phase, the SendingUE adjusts its transmission power to $P_{\text {ini }}$ and sends an ODMA service request carrying $P_{\text {ini }}$ to Node B. Node B can predict $P_{\mathrm{opt}}$ and $N_{\mathrm{opt}}$ based on $P_{\mathrm{ini}}$. By using the predicted $P_{\mathrm{opt}}$ and $N_{\mathrm{opt}}$, Node B checks whether the ODMA request is attainable or not. For nonattainable ODMA requests, Node B simply terminates the PER procedure by replying a rejection message to the SendingUE. For attainable ODMA requests, Node B further derives $P_{\mathrm{TX}}$ RDP and $N_{\max }$, and sends a confirmation message carrying $P_{\mathrm{TX} \_\mathrm{RDP}}$ and $N_{\max }$ to the SendingUE. In the path discovery phase, similar to DSR [11], the SendingUE broadcasts an RREQ through the $i$ th path to Node B to collect $P_{\text {total }, i}$. In this phase, each BackerUE floods the RREQ with transmission power $P_{\mathrm{TX}} \_$RDP and discards the RREQ that exceeds the hop count limitation $N_{\text {max }}$. Based on the collected $P_{\text {total }, i}$, Node B can identify the minimum-power path. As an option, Node B may refuse the ODMA request if $\min _{i} P_{\text {total }, i} \gg P_{\text {opt }}$. In the "path setup phase," Node B sends an RREP packet along the identified path to configure the RelayUEs. 


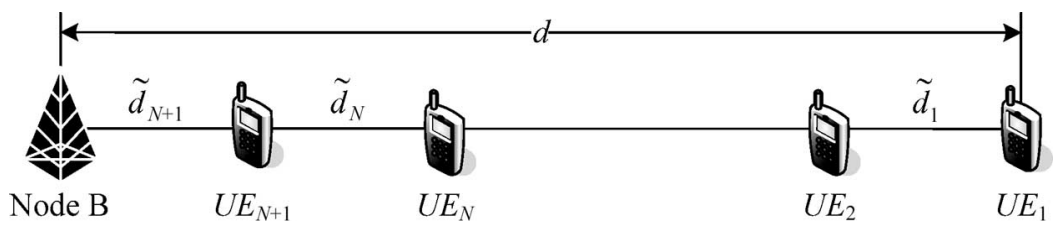

Fig. 1. Colinear network topology consisting of $N+2$ colinear nodes, $U E_{1}, \ldots, U E_{N+1}$, and Node B.

Note that the proposed PER differs from DSR in the following respects. First, PER can predict $P_{\text {opt }}$ before the route discovery. Second, the transmission power utilized to forward the RREQ is $\alpha P_{\text {ref }}$ in DSR but is $P_{\text {TX_RDP }}$ in PER $\left(\alpha P_{\text {ref }} \gg\right.$ $\left.P_{\text {TX_RDP }}\right)$. Third, the hop count limitation for RREQ is infinite in DSR but is $N_{\max }$ in PER. The derivations of $P_{\mathrm{TX} \_\mathrm{RDP}}$, $N_{\text {max }}$, and $P_{\text {ini }}$ are elucidated next.

We investigate $P_{\text {opt }}$ by considering a colinear network topology as shown in Fig. 1, where Node B, $N$ RelayUEs, and SendingUE (i.e., $U E_{1}$ ) are located along a line. For the sake of simplicity, RelayUEs are numbered in order and denoted as $U E_{j}$, where $j=2, \ldots, N+1$. Let $d$ be the distance between SendingUE and Node B. Assume that the UE density in a cell is sufficiently high such that a UE can be found at any location along the line. Let the continuous random number $\tilde{d}_{j}$ denote the distance between $U E_{j}$ and $U E_{j+1}$. During ODMA communication, the UEs are operating in TX and RX modes. In TX mode, the transmission power required by a UE depends on the radio channel condition. Typically, the radio channel condition is characterized by a large-scale propagation model ${ }^{1}$ and a small-scale propagation model ${ }^{2}$ [14]. Rodoplu and Meng [8] have proven that a minimum-power network design is fundamentally the same as that which considers only the path loss. Hence, only a path loss propagation model was considered in the analysis and simulation. The path loss model with the following parameters is used herein: a power law attenuation factor $n(4 \geq n \geq 2)$, antenna gain of a UE's transmitter (receiver) $G_{t}\left(G_{r}\right)$, the wavelength of the modulated signal $\lambda$, the system loss factor $L(L \geq 1)$ [14], and the power required by the UE to correctly decode a message $P_{d}$. Note that $P_{d}$ could be properly set by considering the effects of shadowing and fast fading in the implementation. With the characteristics, we have the following lemma.

\footnotetext{
${ }^{1} \mathrm{~A}$ large-scale propagation model is utilized to predict the mean signal power for a relatively long transmitter-receiver separation. The path loss and the shadowing effect are considered.

${ }^{2}$ Small-scale propagation model characterizing the rapid fluctuations of the received signal strength over a very short distance. Delay spread due to multipath and Doppler effects is considered.
}

Lemma 1: The total power $\left(P_{\mathrm{opt}}\right)$ and the number of RelayUEs $\left(N_{\text {opt }}\right)$ required in the optimal ODMA path are shown in (1) and (2), respectively, at the bottom of the page. In (1) and (2), $k=\left((4 \pi)^{2} L / G_{t} G_{r} \lambda^{2}\right) P_{d}$.

Proof: Denote $P_{\mathrm{TX}, j}$ and $P_{\mathrm{RX}, j}$ as the powers of $U E_{j}$ in TX mode (where $\alpha P_{\text {ref }} \geq P_{\mathrm{TX}, j} \geq P_{\text {ref }}$ ) and RX mode (where $\left.P_{\mathrm{RX}, j}=\beta P_{\mathrm{ref}}\right)$, respectively. The power transmitted by $U E_{j}$ and received by $U E_{j+1}$, denoted as $P_{r, j+1}\left(\tilde{d}_{j}\right)$, is obtained by applying the Friis free space equation [14]

$$
P_{r, j+1}\left(\tilde{d}_{j}\right)=\frac{P_{\mathrm{TX}, j} G_{t} G_{r} \lambda^{2}}{(4 \pi)^{2} \tilde{d}_{j}^{n} L} \triangleq \frac{1}{k_{0}} P_{\mathrm{TX}, j} \tilde{d}_{j}^{-n}
$$

where $k_{0}=(4 \pi)^{2} L / G_{t} G_{r} \lambda^{2}$. For successful reception, $P_{r, j+1}\left(\tilde{d}_{j}\right)$ should not be less than $P_{d}$. Hence

$$
P_{\mathrm{TX}, j}=k_{0} \tilde{d}_{j}^{n} P_{r, j+1}\left(\tilde{d}_{j}\right) \geq k_{0} \tilde{d}_{j}^{n} P_{d} \triangleq k \tilde{d}_{j}^{n}
$$

where $k=k_{0} P_{d}$. The variable $P_{\text {total }, i}$ is obtained by summing the power required by all transmitters and receivers of the SendingUE and RelayUEs. That is,

$$
\begin{aligned}
P_{\mathrm{total}, i} & =\sum_{j=1}^{N+1} P_{\mathrm{TX}, j}+\sum_{j=2}^{N+1} P_{\mathrm{RX}, j} \\
& = \begin{cases}\sum_{j=1}^{N+1} k \tilde{d}_{j}^{n}+N \beta P_{\mathrm{ref}}, & \text { for } k \tilde{d}_{j}^{n}>P_{\mathrm{ref}} \\
(N+1) P_{\mathrm{ref}}+N \beta P_{\mathrm{ref}}, & \text { for } P_{\mathrm{ref}} \geq k \tilde{d}_{j}^{n} .\end{cases}
\end{aligned}
$$

First, we consider the case that $P_{\mathrm{TX}, j}>P_{\text {ref }}$, that is,

$$
\tilde{d}_{j}>\sqrt{[n]} \frac{P_{\text {ref }}}{k} .
$$

By taking the expectation on both sides of (6) and replacing $E\left[\tilde{d}_{j}\right]$ with $d /(N+1)$, we obtain

$$
d \sqrt[n]{\frac{k}{P_{\text {ref }}}}-1>N \geq 0
$$

$$
\begin{aligned}
& P_{\mathrm{opt}}=\left.\min _{i} P_{\mathrm{total}, i}\right|_{N=N_{\mathrm{opt}}}= \begin{cases}k \frac{d^{n}}{\left(N_{\mathrm{opt}}+1\right)^{n-1}}+N_{\mathrm{opt}} \beta P_{\mathrm{ref}}, & \text { for } 0<N_{\mathrm{opt}}<\sqrt[n]{\frac{k}{P_{\mathrm{ref}}}} d-1 \\
\left(N_{\mathrm{opt}}+1+N_{\mathrm{opt}} \beta\right) P_{\mathrm{ref}}, & \text { for } N_{\mathrm{opt}} \geq \sqrt[n]{\frac{k}{P_{\mathrm{ref}}}} d-1\end{cases} \\
& N_{\mathrm{opt}}= \begin{cases}\left\lfloor\sqrt[n]{\frac{k}{P_{\mathrm{ref}}}} d-1,\right\rfloor, & \text { if }\left(\left\lceil\sqrt[n]{\frac{k}{P_{\mathrm{ref}}}} d-1\right]+\beta\right) P_{\mathrm{ref}}>\frac{k d^{n}}{\left\lfloor\sqrt[n]{\frac{k}{P_{\mathrm{ref}}}} d\right\rfloor^{n-1}} \\
\left\lceil\sqrt[n]{\frac{k}{P_{\mathrm{ref}}}} d-1\right], & \text { otherwise }\end{cases}
\end{aligned}
$$


From (5), $P_{\text {total }, i}$ is derived by

$$
\begin{array}{r}
P_{\text {total }, i}=k\left(\sum_{j=1}^{N} \tilde{d}_{j}^{n}+\left(d-\sum_{j=1}^{N} \tilde{d}_{j}\right)^{n}+N \beta P_{\text {ref }}\right. \\
\text { for } d \sqrt[n]{\frac{k}{P_{\text {ref }}}}-1>N \geq 0 .
\end{array}
$$

The lower bound of (8) is obtained by varying $\tilde{d}_{j}$, that is,

$$
\min _{i} P_{\text {total }, i}=\left.P_{\text {total }, i}\right|_{\tilde{d}_{j}=\bar{d}_{j}, \quad \text { for } j=1, \ldots, N+1} .
$$

The optimal distance between adjacent nodes $\bar{d}_{j}$ that results in the minimum $P_{\text {total }, i}$ is obtained by $\left(\partial / \partial \tilde{d}_{j}\right) P_{\text {total }, i}=0$, for $j=1, \ldots, N+1$. Thus

$$
\bar{d}_{1}=\bar{d}_{2}=\cdots=\bar{d}_{N+1}=d-\sum_{i=1}^{N} \bar{d}_{i}=\frac{d}{N+1} .
$$

Equation (10) demonstrates that, for a given $N$, the lower bound is achieved if the distances between any two adjacent RelayUEs are equal. Under this condition, the transmission power required by each RelayUE to reach its neighboring RelayUE is a constant, which is denoted as $P_{0}$, where

$$
P_{0}=\left.k \tilde{d}_{i}^{n}\right|_{\tilde{d}_{i}=\bar{d}_{i}}=k\left(\frac{d}{N+1}\right)^{n} .
$$

From (8) and (11), $\min _{i} P_{\text {total }, i}$ is obtained by

$$
\begin{aligned}
\min _{i} P_{\text {total }, i}= & (N+1) P_{0}+N \beta P_{\text {ref }} \\
= & \left(k \frac{d^{n}}{(N+1)^{n-1}}+N \beta P_{\text {ref }}\right) \\
& \text { for } 0<N<\sqrt[n]{\frac{k}{P_{\text {ref }}}} d-1 .
\end{aligned}
$$

Equation (12) is a monotonically decreasing function of $N$ because $(d / d N) P_{t}<0$, for $n \geq 2$ and $N<\sqrt[n]{\left(k / P_{\text {ref }}\right)} d-1$. Since $N$ should be an integer, $P_{\text {opt }}$ is obtained when

$$
N=N_{\text {opt }}=\left\lfloor\sqrt[n]{\frac{k}{P_{\text {ref }}}} d-1\right\rfloor
$$

Now consider the case that $P_{\mathrm{TX}, j} \leq P_{\text {ref }}$, or equivalently, $N \geq$ $\sqrt[n]{k / P_{\text {ref }}} d-1$. From (5), $\min _{i} P_{\text {total }, i}$ is obtained by

$$
\begin{aligned}
\min _{i} P_{\text {total }, i}=(N+1+N \beta) & P_{\text {ref }} \\
& \text { for } \quad N \geq \sqrt[n]{\frac{k}{P_{\text {ref }}}} d-1 .
\end{aligned}
$$

Equation (14) is a monotonically increasing function of $N$. Since $N$ should be an integer, $P_{\text {opt }}$ is located when

$$
N=N_{\text {opt }}=\left\lceil\sqrt[n]{\frac{k}{P_{\text {ref }}}} d-1\right\rceil .
$$

Combining (13) and (15), we obtain (2). And, $P_{\text {opt }}$ given in (1) is obtained by combining (12) and (14).
A similar result of Lemma 1 has been obtained in [15]. Lemma 1 indicates that $P_{t}$ and $P_{\text {opt }}$ depend on following parameters: UE capabilities (i.e., $\beta, P_{\text {ref }}, P_{d}$ ), the path loss exponent (i.e., $n$ ), the distance between a source UE and Node $\mathrm{B}$ (i.e., $d$ ), and the number of RelayUEs (i.e., $N$ ). Among these parameters, the only unknown factor is $d$. In mobile cellular networks, UE normally utilizes an open-loop power control mechanism [14] to estimate $d$. Let $P_{\mathrm{BCH}}$ and $P_{\mathrm{avg}}$ be the broadcast channel $(\mathrm{BCH})$ power transmitted by Node $\mathrm{B}$ and the average power received by SendingUE, respectively. In UMTS, $P_{\mathrm{BCH}}$ is a constant and is periodically broadcasted by Node B. Hence, SendingUE can estimate $d$ from (4), that is,

$$
d=\sqrt[n]{\frac{P_{\mathrm{BCH}}}{k P_{\mathrm{avg}}}}
$$

From (4) and (16), the initial transmission power used by SendingUE to send the ODMA service request to Node B $P_{\text {ini }}$ is given by

$$
P_{\text {ini }}=k d^{n}=\frac{P_{\mathrm{BCH}}}{P_{\mathrm{avg}}} .
$$

Lemma 1: suggests that, with $N_{\max }=N_{\text {opt }}+1$ and $P_{\text {TX_RDP }}=P_{0}=k\left(d / N_{\text {max }}\right)^{n}$, an optimal path in a colinear network topology is obtained given sufficiently high UE density. For a cellular network with low UE density, the optimal path may not be found. To solve this problem, RelayUE must increase $P_{\mathrm{TX}}$ RDP to find another RelayUE in its neighborhood. Therefore, the minimum-power path can still be obtained

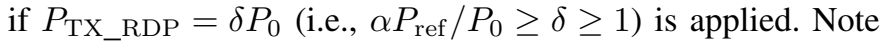
that, under this condition, the total power required by the minimum-power path is not less than $P_{\mathrm{opt}}$.

Normally, RelayUEs are located between SendingUE and Node B. As demonstrated in Fig. 2, BackerUEs in the region where the two circles overlap (both solid circles centered at Node B and SendingUE have the same radius $P_{\text {ini }}$ ) could be possible RelayUE candidates. Hence, in PER, only these BackerUEs, rather than all BackerUEs in the entire cell, should forward RREQ during route discovery. These BackerUEs can be identified easily because they can receive both the ODMA service request and the confirmation from SendingUE and Node B, respectively.

Figs. 2 and 3 show a general network topology and the message flows employed to demonstrate a scenario of the PER mechanism, respectively. In this scenario, $U E_{1}$ is the SendingUE, $U E_{j}$ s, for $j=2, \ldots, 12$, are BackerUEs, and $N_{\text {opt }}=1$ is assumed. As shown in Fig. 2, $U E_{11}$ cannot receive the ODMA service request from $U E_{1}$, and $U E_{12}$ cannot receive the confirmation from Node $\mathrm{B}$; hence, $U E_{11}$ and $U E_{12}$ automatically enter the SLP mode after timeout. The RREQ message traversing along $U E_{1}-U E_{6}-U E_{7}$ is discarded by $U E_{7}$ because $N_{\max }$ is reached. Not otherwise specified, messages are carried through the logical channels specified in parenthesis in Fig. 3 (i.e., ORACH denotes the ODMA random access channel [2]). The three phases of the PER mechanism are described as follows. 


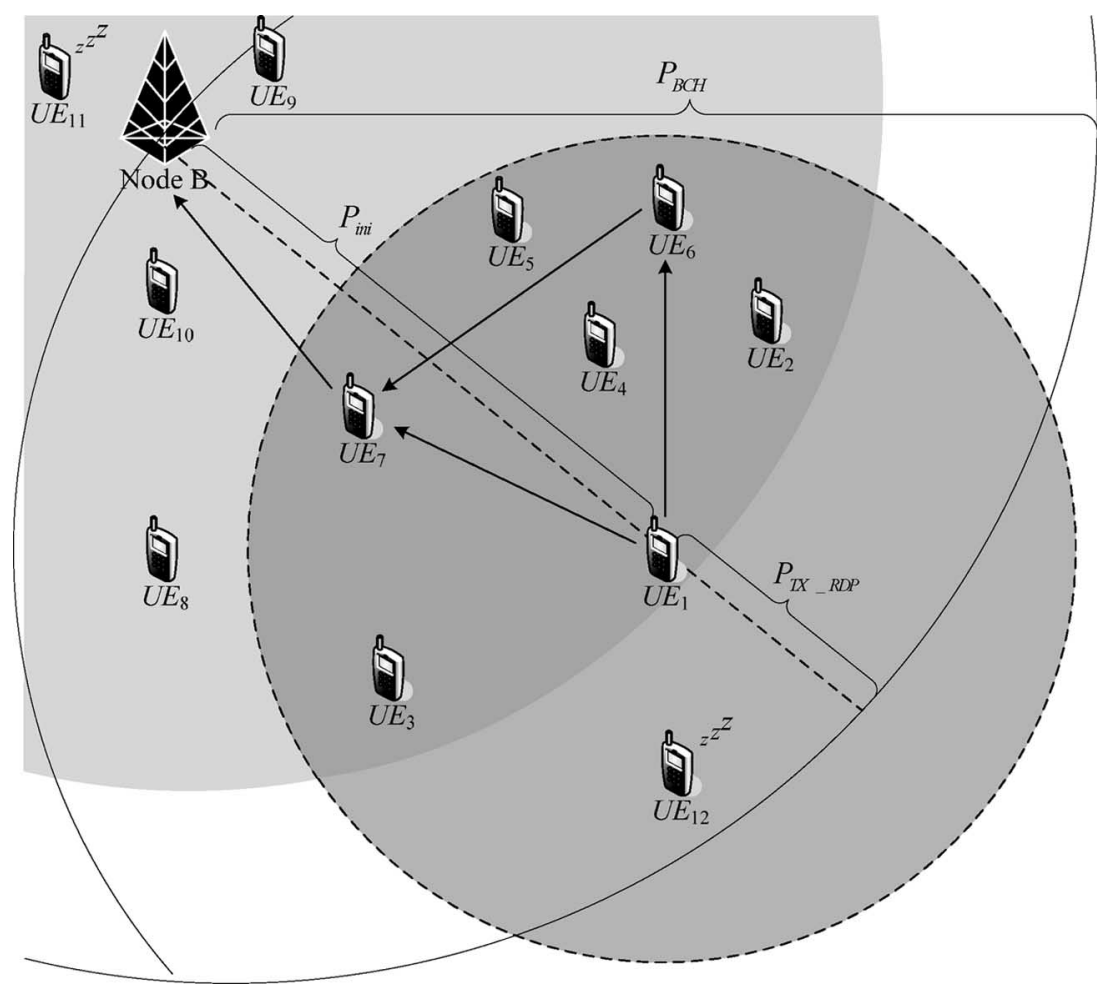

Fig. 2. Network topology illustrating the PER mechanism.

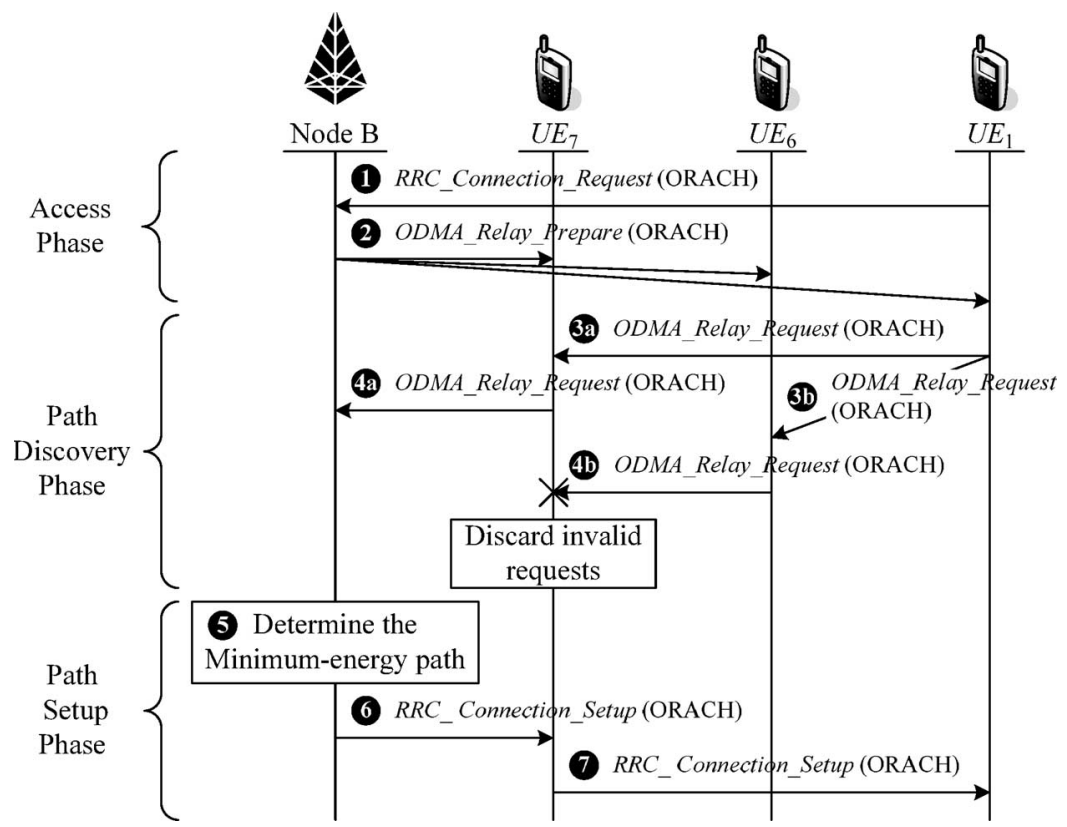

Fig. 3. Message flow of the PER mechanism.

\section{A. Access Phase}

Step 1) Prior to communicating with Node $B$, the SendingUE $U E_{1}$ measures $P_{\text {avg, }}$, adjusts its transmission power to $P_{\text {ini }}$, and then sends $\mathrm{RRC}_{-}$ Connection_Req [2] carrying $P_{\text {ini }}$ to Node B.

Step 2) Upon receiving the RRC_Connection_Req message, Node $B$ rejects the request if the request is nonattainable. Otherwise, Node B derives $P_{\mathrm{TX}}$ RDP and $N_{\text {max }}$, adjusts its transmission power to $P_{\text {ini }}$, and acknowledges ODMA_Relay_Prepare carrying $P_{\mathrm{TX} \_ \text {RDP }}$ and $N_{\max }$ to $U E_{1}$.

\section{B. Path Discovery Phase}

In the path discovery phase, the SendingUE adjusts its transmission power to $P_{\mathrm{TX}}$ RDP and floods an RREQ (i.e., 


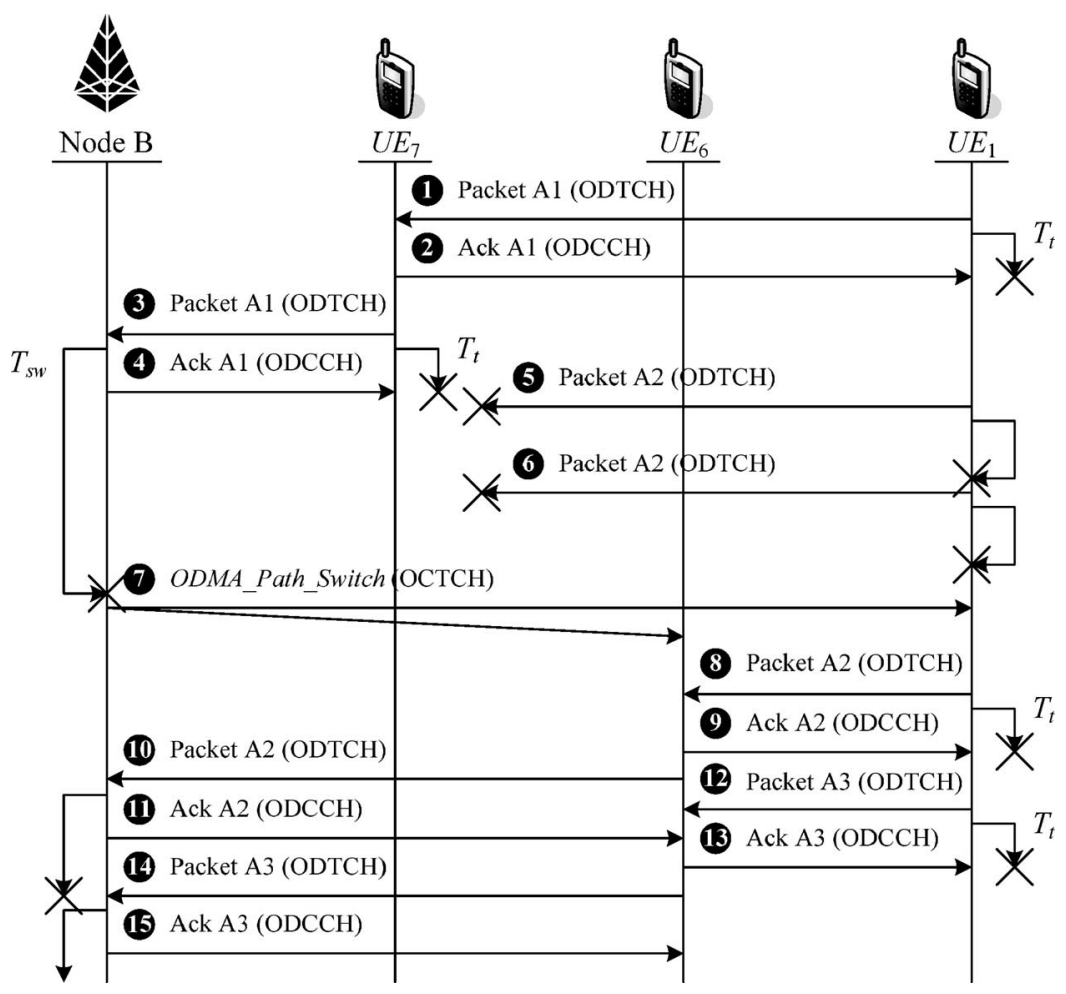

Fig. 4. Message flow of the PER mechanism link management functions.

ODMA_Relay_Req) the surrounding BackerUEs. The RREQ carries three parameters: namely 1) SID; 2) RoutingList; and 3) $P_{\text {acc, } j}$. The $S I D$ is the ODMA_ID of the SendingUE and is utilized to identify a specific ODMA connection request; the RoutingList contains ODMA_IDs of UEs that comprise the specific path; and the $P_{\text {acc, } j}$ is the accumulated power required for the path from SendingUE to $U E_{j}$.

Step 3a) $U E_{1}$ sends ODMA_Relay_Req carrying $(S I D=$ 1, RoutingList $=$ NULL, $\left.\quad P_{\mathrm{acc}, 1}=0\right)$ to its neighboring UEs, and $U E_{7}$ updates the accumulated power by

$$
\begin{aligned}
P_{\mathrm{acc}, 7} & =P_{\mathrm{acc}, 1}+P_{\mathrm{TX}, 1}+P_{\mathrm{RX}, 7} \\
& =P_{\mathrm{acc}, 1}+\max \left(P_{\mathrm{TX} \_\mathrm{RDP}}-P_{r, 7}, P_{\mathrm{ref}}\right)+\beta P_{\mathrm{ref}}
\end{aligned}
$$

where $P_{r, 7}$ is the power of $U E_{1}$ 's ODMA_ Relay_Req measured at $U E_{7}$.

Step 4a) $U E_{7}$ forwards the RREQ carrying $(S I D=1$, RoutingList $\left.=7, P_{\mathrm{acc}, 7}\right)$ to Node B. Node B updates the total power $P_{\text {total }, i}$ of this first path by

$$
\begin{aligned}
P_{\text {total }, 1} & =P_{\mathrm{acc}, 7}+P_{\mathrm{TX}, 7} \\
& =P_{\mathrm{acc}, 7}+\max \left(P_{\mathrm{TX} \_\mathrm{RDP}}-P_{r, \text { NodeB }}, P_{\text {ref }}\right)
\end{aligned}
$$

where $P_{r, \text { NodeB }}$ is the power of $U E_{7}$ 's ODMA_Relay_Req measured at Node B. Note that the power used by Node B's receiver is a common factor for all paths and thus is not considered in calculating $P_{\text {total }, i}$.
Step 3b) $U E_{6}$ receives the RREQ from $U E_{1}$, updates the triplet for this second path, and forwards the RREQ to $U E_{7}$.

Step 4b) $U E_{7}$ discards the RREQ because $N_{\max }$ is reached.

\section{Path Setup Phase}

Step 5) Node B determines the minimum-power path, which has the least $P_{\text {total }, i}$ among all discovered paths, and identifies $U E_{7}$ as the RelayUE from the RoutingList.

Step 6) Node B sends an RRC_Connection_Setup [2] to $U E_{7}$ carrying the ODMA traffic channel (ODTCH) and ODMA control channel (ODCCH) allocations [2]. The remaining BackerUEs whose ODMA_ID are not on the RoutingList move to the SLP mode.

Step 7) The ODMA communication path is established. The established communication path may be broken if mobility UEs are further considered. In such a mobile environment, Node B may repeat Steps 5) to 7) to create one or more backup communication paths to the Sending UE and enable link management functions for managing these paths. In the implementation, PER employs a well-known sliding-window scheme with a stop-and-wait automatic retransmission request (ARQ) mechanism to control data flow and retransmit error packets between adjacent RelayUEs. The same network topology shown in Fig. 2 is utilized to demonstrate a scenario of link management functions. In this scenario, $U E_{1}-U E_{7}-$ NodeB is the primary path and $U E_{1}-U E_{6}-$ NodeB is the backup path. Fig. 4 shows 
the message flow of the link management functions. In this scenario, the sliding-window size $W_{\max }$ is 2 , and timers $T_{t}$ and $T_{s w}$ are required by UEs to control packet retransmission and by Node B to control path switching, respectively.

\section{Flow Control and Error Control}

Step 1) $U E_{1}$ starts its $T_{t}$ and transmits packet A1 through the primary path to $U E_{7}$.

Step 2) $U E_{7}$ sends an acknowledgment to $U E_{1}$ denoting the successful reception of $\mathrm{A} 1$; then, $U E_{1}$ resets and stops its $T_{t}$.

Step 3) $U E_{7}$ starts its $T_{t}$ and forwards A1 to Node B. Node B resets and starts $T_{s w}$ whenever it correctly receives a new packet.

Step 4) $U E_{7}$ resets and stops its $T_{t}$ after receiving the acknowledgment that $\mathrm{A} 1$ was received.

Step 5) $U E_{1}$ starts its $T_{t}$ and transmits a packet $\mathrm{A} 2$ to $U E_{7}$. A2 is lost.

Step 6) $U E_{1}$ retransmits A2 after the expiry of its $T_{t}$. A2 is lost and $T_{t}$ expires again. Since A2 has transmitted $W_{\max }$ times, the retransmission is stopped.

\section{E. Switch to the Backup Path}

Step 7) After $T_{s w}$ expires, Node B switches to the backup path by sending ODMA_Path_Switch to $U E_{6}$ and $U E_{1}$ to activate the backup path.

Step 8) $U E_{1}$ starts its $T_{t}$ and transmits the last unacknowledged packet $\mathrm{A} 2$ over the activated path to $U E_{6}$.

Step 9) $U E_{1}$ resets and stops $T_{t}$ after receiving an acknowledgment from $U E_{6}$.

Step 10) $U E_{6}$ starts its $T_{t}$ and forwards A2 to Node B; Node B resets and starts its $T_{s w}$ after receiving a packet from $U E_{6}$.

Step 11) $U E_{6}$ resets and stops its $T_{t}$ after receiving an acknowledgment from Node $B$.

Steps 12)-15) $U E_{1}$ successfully transmits A3 to Node B.

\section{NUMERICAL RESULTS}

Simulations were conducted to verify the effectiveness of the proposed PER mechanism. The load balancing capability of ODMA was not investigated herein. Hence, a single cell with 50-500 nonmobile UEs was considered. All UEs were assumed to be uniformly distributed within a hexagonal cell with radius $2500 \mathrm{~m}$. The constants used herein are listed as follows: $\lambda=15.78 \mathrm{~cm}, G_{t}=G_{r}=1, L=1, k_{0}=6334, \alpha=20$, $\beta=0.1, \ldots, 0.9, n=2, P_{\text {ref }}=20 \mathrm{~mW}, P_{d}=10^{-8} \mathrm{~mW}, d=$ $2100 \mathrm{~m}$, and $\delta=2$. Each sample during the simulation was obtained by averaging the outcomes from $10^{6}$ identical experiments. Both DSR and PER were simulated. The DSR was chosen as a benchmark because it can explore all paths and identify the minimum-power path in a cell. In the simulation, both DSR and PER found the same minimum-power path,

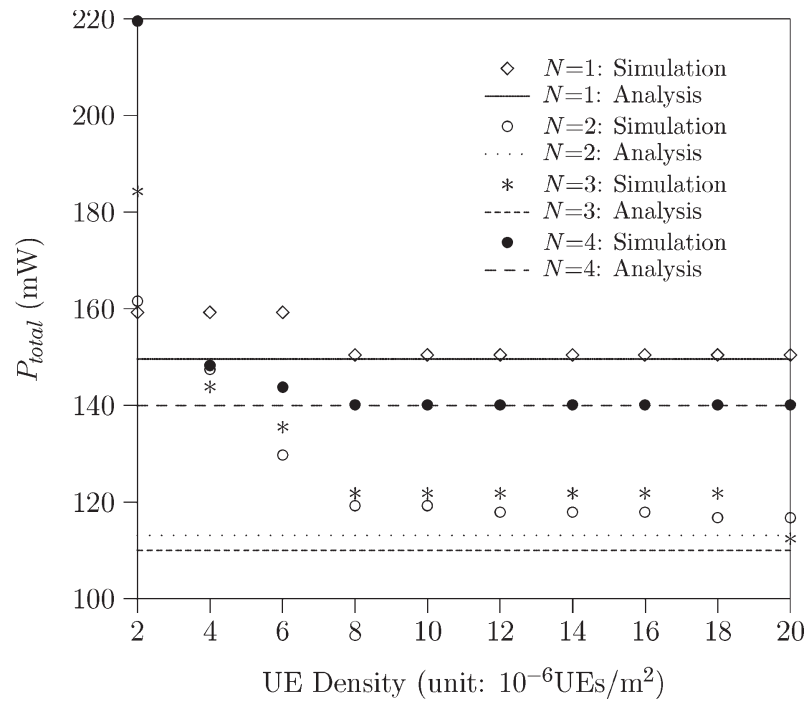

Fig. 5. Total power required by the path for various UE densities and $N$.

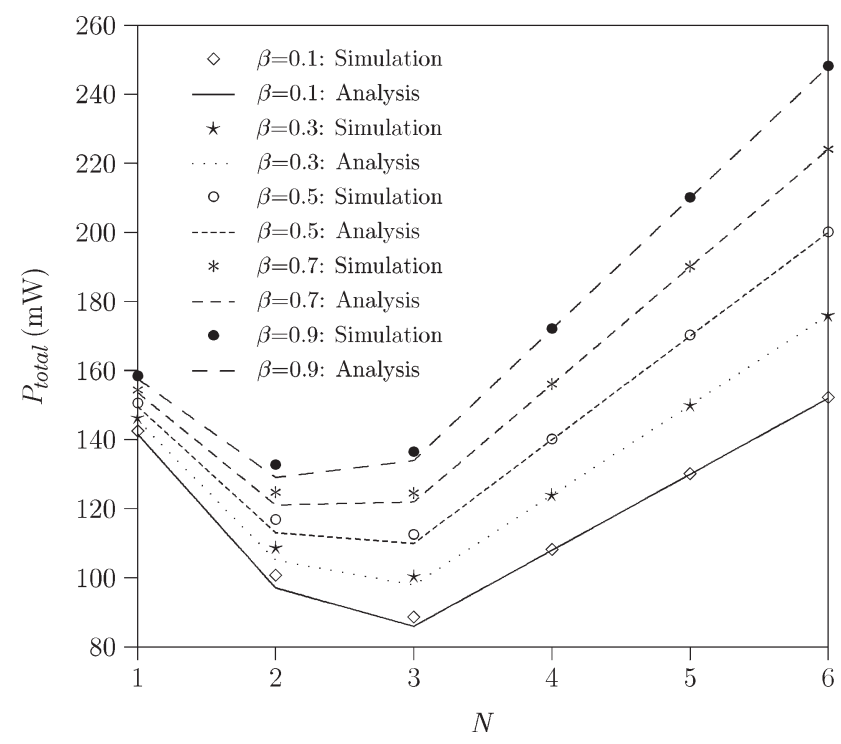

Fig. 6. Total power required by the path for various $N$ and $\beta$.

but with different signaling overheads. Hence, the minimumpower path discovered by DSR was not specifically identified in Figs. 5 and 6. In Figs. 5 and 6, the analytical results are denoted with lines, while the simulation results are presented with symbols.

The accuracy of the analysis was first verified by simulation. In Fig. 5, the total power required by the minimum-power path (i.e., $P_{\text {total }}$, where $P_{\text {total }} \simeq \min _{i} P_{\text {total }, i}$ ) for various UE densities and the number of RelayUEs (i.e., $N$ ) were shown, in which $\beta=0.5$ was assumed. Lemma 1 obtained $N_{\mathrm{opt}}=3$ and $P_{\mathrm{opt}}=110 \mathrm{~mW}$. Note that for $d=2100 \mathrm{~m}$, SendingUE required $279 \mathrm{~mW}$ to transmit data directly to Node B without using ODMA. Simulation results showed estimation errors for low UE densities (Fig. 5). However, the estimation error was considerably reduced when the UE density was larger than $5 \times 10^{-6} \mathrm{UEs} / \mathrm{m}^{2}$. This finding was a result of the high UE density assumption in Lemma 1. For low UE density, RelayUEs 


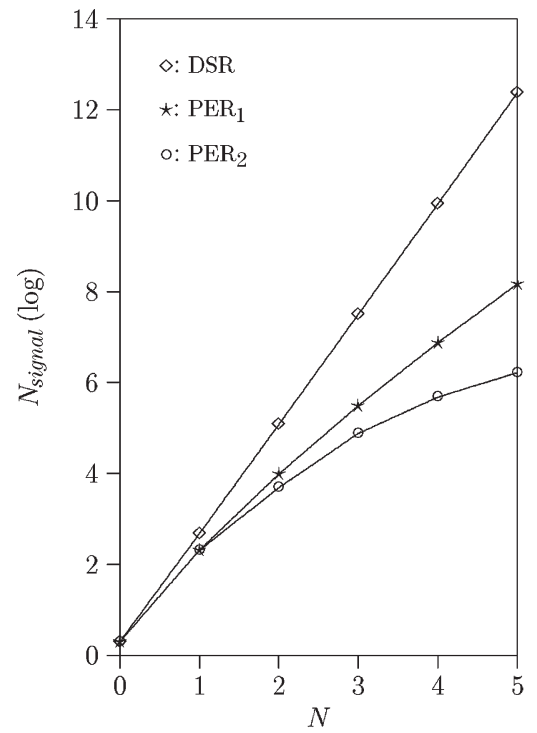

(a)

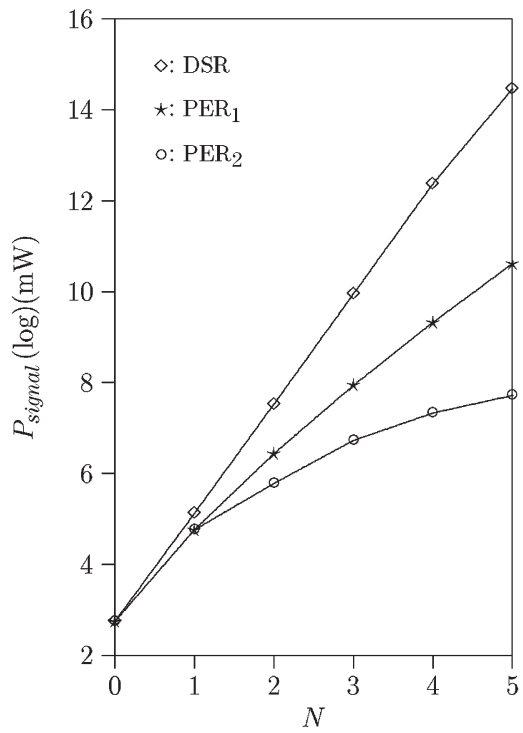

(b)

Fig. 7. Signaling cost of DSR and PER. (a) Total number of RREQ messages. (b) Total power consumed by RREQ messages.

could not be found at expected locations, and therefore, the lower bound was not achieved.

In the following two examples, the UE density is fixed to $2 \times 10^{-5} \mathrm{UEs} / \mathrm{m}^{2}$. Fig. 6 showed $P_{\text {total }}$ for various $N \mathrm{~s}$ and $\beta \mathrm{s}$. From Lemma 1 , it can be derived that $N_{\mathrm{opt}}=2$ for $\beta=0.7$ and $\beta=0.9$, and $N_{\mathrm{opt}}=3$ for $\beta=0.1, \beta=0.3$, and $\beta=0.5$; each derived $N_{\text {opt }}$ coincided with the simulation results shown in Fig. 6. Fig. 6 demonstrated that for a fixed $N$, a decreased $\beta$ resulted in a lowered $P_{\text {total }}$ since a low power is required by the receiver of each RelayUE. For a given $\beta, P_{\text {total }}$ was first decreased and then increased when $N$ was increased from 1 to 6 . The rationale for the variation of $P_{\text {total }}$ is described as follows. Increasing $N$ meant to add new RelayUEs in the path. Since these new RelayUEs consume extra power, it is not valuable to reduce $P_{\text {total }}$ by increasing the number of RelayUEs unlimitedly, particularly for those RelayUEs that have high $\beta$. In other words, using RelayUEs closer than $1 /\left(N_{\text {opt }}+1\right)$ together results in greater overall power consumption since the savings in TX power from using smaller hops is lost given that nothing less than $P_{\text {ref }}$ can be used. Lemma 1 proved that the minimum $P_{\text {total }}$ was obtained if $N_{\text {opt }}$ RelayUE was utilized in a path. For $N<N_{\text {opt }}$, increasing $N$ implied a decrease in the distance between two adjacent RelayUEs; hence, the transmission power of existing RelayUEs was reduced. However, the cost was the extra power consumption introduced by new RelayUEs. In the region of $N<N_{\text {opt }}, P_{t}$ was decreased because the power required by new RelayUEs is less than the power reduced by existing RelayUEs. However, in the region of $N>N_{\text {opt }}$, reducing the distance between two adjacent RelayUEs did not further reduce the transmission power of each RelayUE because the transmission power was bounded by $P_{\text {ref }}$; therefore, $P_{\text {total }}$ was monotonically increased.

As mentioned earlier, both DSR and PER were able to locate the same minimum-power path; however, their signal costs were substantially different. In DSR, the UEs flood the RREQ over the entire cell with transmission power $\alpha P_{\text {ref }}$.
However, in PER, only selected BackerUEs flood the RREQ with transmission power $\delta P_{0}$. Fig. 7 shows the signaling cost of DSR and PER. The number of RREQs, (i.e., denoted as $N_{\text {signal }}$ ) and the total power consumed by the RREQs (i.e., denoted as $\left.P_{\text {signal }}\right)$ were investigated and illustrated in Fig. 7(a) and (b), respectively. In this example, $\beta=0.5$, and $\delta=\alpha P_{\text {ref }} / P_{0}$ and $\delta=2$ were used in $\mathrm{PER}_{1}$ and $\mathrm{PER}_{2}$, respectively. That is, both $\mathrm{PER}_{1}$ and DSR used UE's maximum transmission power to flood the RREQ. As shown in the figures, the proposed PER mechanism dramatically reduced $N_{\text {signal }}$ and $P_{\text {signal }}$ because, in PER, fewer BackerUEs were allowed to forward the RREQ. The figures also demonstrated that a small $\delta$ results in a small $N_{\text {signal }}$ and $P_{\text {signal }}$. However, reducing $N_{\text {signal }}$ by lowering $\delta$ increased the risk of locating no path during the route discovery, particularly for those networks with low UE density. Since the optimization of $\delta$ is not essential for the effectiveness of the PER mechanism, its optimization will be the subject of future work.

\section{CONCLUSION}

This paper presents a PER mechanism for ODMA cellular networks. In contrast to previous routing approaches, the proposed PER mechanism can estimate the power consumption of, and the number of relay nodes for, an optimal path without information from the other nodes. With the estimation, route discovery procedures originating from nonattainable ODMA requests can be prevented. The PER mechanism further provides attainable ODMA requests, a method to set the transmission power and maximum hop count to reduce the power consumption of each UE during the route discovery. The effectiveness of the proposed method is shown both theoretically and via simulation. Simulation results demonstrate that, with carefully chosen parameters, the PER mechanism can identify the minimum-power path with relatively low signaling cost compared to that of DSR. 
TABLE I

PARAMETER DEFINITION

\begin{tabular}{|c||c|}
\hline \multicolumn{1}{|c||}{ Parameters } & Descriptions \\
\hline \hline$N$ & Number of RelayUE in the collinear model \\
\hline$P_{d}$ & The power required by the UE to correctly decode a message \\
\hline$d$ & Distance between the Sending $U E$ and Node B \\
\hline$P_{T X, j}$ & Transmission power of $U E_{j}$ in TX mode, $\alpha P_{r e f} \geq P_{T X, j} \geq P_{r e f}$ \\
\hline$P_{R X, j}$ & Receiving power of $U E_{j}$ in RX mode, $P_{R X, j}=\beta P_{r e f}$ \\
\hline$P_{r, j+1}\left(\tilde{d}_{j}\right)$ & The power transmitted by $U E_{j}$ and received by $U E_{j+1}$ \\
\hline$n$ & The power-law attenuation factor \\
\hline$G_{t}$ & The antenna gain of an UE's transmitter \\
\hline$G_{r}$ & The antenna gain of an UE's receiver \\
\hline$L$ & The system-loss factor $(L \geq 1)$ \\
\hline$\lambda$ & Wavelength of the modulated signal \\
\hline
\end{tabular}

\section{APPENDIX}

The definition of parameters involved in the analysis is summarized in Table I.

\section{ACKNOWLEDGMENT}

The authors would like to thank anonymous reviewers for their valuable comments that helped improve the quality of the paper.

\section{REFERENCES}

[1] H. Karl, "An overview of energy-efficiency techniques for mobile communication systems," Telecommunication Networks Group, Technical University Berlin, Berlin, Germany, Tech. Rep. TKN-03-017, Oct. 2003. AG Mobikom WG7.

[2] "Opportunity Driven Multiple Access," Tech. Rep. 25.924, Dec. 1999. 3GPP, version 1.0.0.

[3] T. Rouse, I. Band, and S. McLaughlin, "Congestion-based routing strategies in multihop TDD-CDMA networks," IEEE J. Sel. Areas Commun., vol. 23, no. 3, pp. 668-681, Mar. 2005.

[4] P. Lin, W.-R. Lai, and C.-H. Gan, "Modeling opportunity driven multiple access in UMTS," IEEE Trans. Wireless Commun., vol. 3, no. 5, pp. 1669-1677, Sep. 2004.

[5] A. J. Goldsmith and S. B. Wicker, "Design challenges for energyconstrained ad hoc wireless networks," IEEE Wirel. Commun., vol. 9, no. 4, pp. 8-27, Aug. 2002.

[6] S. Singh, M. Woo, and C. S. Raghavendra, "Power-aware routing in mobile ad hoc networks," in Proc. 4th Annu. ACM/IEEE Int. Conf. Mobile Comput. and Netw. (MobiCom), 1998, pp. 181-190.

[7] J.-H. Chang and L. Tassiulas, "Energy conserving routing in wireless ad-hoc networks," in Proc. IEEE 19th Annu. Joint Conf. IEEE Comput. and Commun. Soc. (INFOCOM), 2000, vol. 1, pp. 22-31.

[8] V. Rodoplu and T. H. Meng, "Minimum energy mobile wireless networks," IEEE J. Sel. Areas Commun., vol. 17, no. 8, pp. 1333-1344, Aug. 1999.

[9] R. Wattenhofer, L. Li, P. Bahl, and Y. M. Wang, "Distributed topology control for power efficient operation in multihop wireless ad hoc networks," in Proc. IEEE 20th Annu. Joint Conf. IEEE Comput. and Commun. Soc. (INFOCOM), 2001, vol. 3, pp. 1388-1397.

[10] "ODMA Routing With Procedures for Mobile Originated Calls, Mobile Terminated Calls, and Location Update," Tech. Rep. Tdoc TSGR2\#2(99) 179, 1999. 3GPP TSG-RAN WG2.

[11] D. Johnson and D. Maltz, "Dynamic source routing in ad hoc wireless networks," in Mobile Computing. Norwell, MA: Kluwer, 1996, pp. $153-181$.

[12] C. E. Perkins, E. M. Belding-Royer, and S. Das, ad hoc On-Demand Distance Vector (AODV) Routing, Jul. 2003. RFC 3561.

[13] A. M. Safwat, H. S. Hassanein, and H. T. Mouftah, "Structured proactive and reactive routing for wireless mobile ad hoc networks," in The Handbook of ad hoc Wireless Networks. Boca Raton, FL: CRC, pp. 233-244.

[14] T. S. Rappaport, Wireless Communications: Principles and Practices, 2nd ed. Englewood Cliffs, NJ: Prentice-Hall, 2002.

[15] M. Bhardwaj, T. Garnett, and A. P. Chandrakasan, "Upper bounds on the lifetime of sensor networks," in Proc. IEEE ICC, 2001, vol. 3, pp. $785-790$.

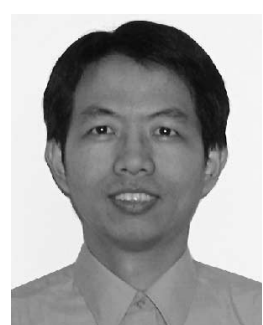

Ray-Guang Cheng (S'94-M'97) received the B.E., M.E., and Ph.D. degrees in communication engineering from the National Chiao Tung University, Hsinchu, Taiwan, R.O.C., in 1991, 1993, and 1996 , respectively.

From 1997 to 2000, he was as a Researcher and a Project Leader with the Advance Technology Center, Computer and Communication Laboratories, Industrial Technology Research Institute (ITRI). He was involved in the designing of medium access control and radio resource management algorithms for GPRS and UMTS systems. His team was named "Top Research Team of the Year" by ITRI and he received the "Outstanding Technology Prize" from Ministry of Economic Affairs in 2000. From 2000 to 2003, he was the Senior Manager of the R\&D Division, BenQ Mobile System Inc. Since 2003, he has been with the Department of Electronic Engineering, National Taiwan University of Science and Technology, Taipei, Taiwan, as an Assistant Professor. His research interests include multihop wireless networks and multimedia communications.

Dr. Cheng is a member of the IEEE Communication Society and the $\mathrm{Ph}$ Tau Phi Scholastic Honor Society. He received the "Best Industrial-based Paper Award" from the Ministry of Education, Taiwan, in 1998.

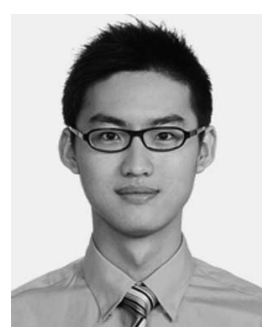

Shin-Ming Cheng received the BSCSIE degree from the National Taiwan University of Science and Technology, Taipei, Taiwan, R.O.C., in 2000. He is currently working toward the Ph.D. degree in the Department of Computer Science and Information Engineering, National Taiwan University of Science and Technology.

His current research interests include mobile computing, personal communications services, and wireless Internet.

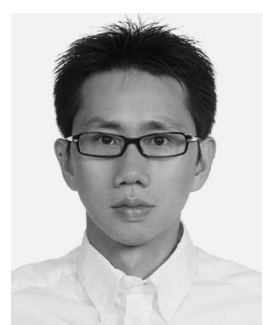

Phone Lin (M'02-SM'06) received the B.S. degree in computer science and information engineering and the Ph.D. degree from the National Chiao Tung University, Hsinchu, Taiwan, R.O.C., in 1996 and 2001, respectively.

From August 2001 to July 2004, he was an Assistant Professor with the Department of Computer Science and Information Engineering (CSIE), National Taiwan University (NTU), Taipei, Taiwan, R.O.C. Since August 2004, he has been an Associate Professor with the Department of CSIE and the Graduate Institute of Networking and Multimedia, NTU. His current research interests include personal communications services, wireless Internet, and performance modeling.

Dr. Lin is an Associate Editor of the IEEE TRANSACTIONS ON VEHICULAR TEChNOLOGY, a Guest Editor of the IEEE TRANSACTIONS ON WiRELESS COMMUNiCATIONS special issue on Mobility and Resource Management, and a Guest Editor for the Association for Computing Machinery/Springer Mobile Networks and Applications special issue on Wireless Broad Access. He is also an Associate Editorial Member for the Wireless Communications and Mobile Computing Journal. He has received many research awards, such as the Young Researchers Award from the Pan Wen-Yuan Foundation in Taiwan in 2004, the K. T. Li Young Researcher Award from the ACM Taipei Chapter in 2004, the Wu Ta You Memorial Award of the National Science Council of Taiwan in 2005, and the Fu Suu-Nien Award from NTU in 2005. He is listed in Who's Who in Science and Engineering in 2006. 